\title{
TU/e EmonOWEN

\section{An analytical force balance model for dust particles with size up to several Debye lengths}

\section{Citation for published version (APA):}

Aussems, D. U. B., Khrapak, S. A., Doğan, I., van de Sanden, M. C. M., \& Morgan, T. W. (2017). An analytical force balance model for dust particles with size up to several Debye lengths. Physics of Plasmas, 24(11), [113702]. https://doi.org/10.1063/1.5001576

DOI:

$10.1063 / 1.5001576$

Document status and date:

Published: 01/11/2017

\section{Document Version:}

Publisher's PDF, also known as Version of Record (includes final page, issue and volume numbers)

\section{Please check the document version of this publication:}

- A submitted manuscript is the version of the article upon submission and before peer-review. There can be important differences between the submitted version and the official published version of record. People interested in the research are advised to contact the author for the final version of the publication, or visit the $\mathrm{DOI}$ to the publisher's website.

- The final author version and the galley proof are versions of the publication after peer review.

- The final published version features the final layout of the paper including the volume, issue and page numbers.

Link to publication

\section{General rights}

Copyright and moral rights for the publications made accessible in the public portal are retained by the authors and/or other copyright owners and it is a condition of accessing publications that users recognise and abide by the legal requirements associated with these rights.

- Users may download and print one copy of any publication from the public portal for the purpose of private study or research.

- You may not further distribute the material or use it for any profit-making activity or commercial gain

- You may freely distribute the URL identifying the publication in the public portal.

If the publication is distributed under the terms of Article 25fa of the Dutch Copyright Act, indicated by the "Taverne" license above, please follow below link for the End User Agreement:

www.tue.nl/taverne

Take down policy

If you believe that this document breaches copyright please contact us at:

openaccess@tue.nl

providing details and we will investigate your claim. 


\section{An analytical force balance model for dust particles with size up to several Debye lengths}

D. U. B. Aussems, S. A. Khrapak, İ. Doğan, M. C. M. van de Sanden, and T. W. Morgan

Citation: Physics of Plasmas 24, 113702 (2017);

View online: https://doi.org/10.1063/1.5001576

View Table of Contents: http://aip.scitation.org/toc/php/24/11

Published by the American Institute of Physics

\section{Articles you may be interested in}

Higher order structure in a complex plasma

Physics of Plasmas 24, 103701 (2017); 10.1063/1.4990510

Effect of magnetic field on the phase transition in a dusty plasma

Physics of Plasmas 24, 113703 (2017); 10.1063/1.5003972

Isothermal equation of state of three dimensional Yukawa gas

Physics of Plasmas 24, 113704 (2017); 10.1063/1.5000409

Parameters of a runaway electron avalanche

Physics of Plasmas 24, 103505 (2017); 10.1063/1.4990729

Computational studies on scattering of radio frequency waves by density filaments in fusion plasmas Physics of Plasmas 24, 102115 (2017); 10.1063/1.4992032

Rogue waves in space dusty plasmas

Physics of Plasmas 24, 113701 (2017); 10.1063/1.5000027

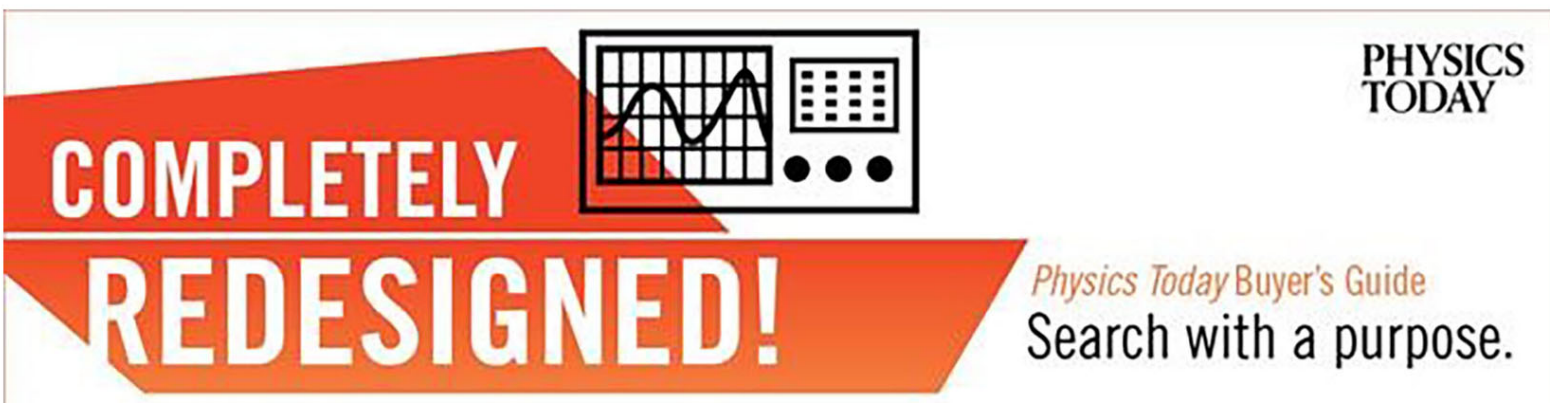




\title{
An analytical force balance model for dust particles with size up to several Debye lengths
}

\author{
D. U. B. Aussems, ${ }^{1}$ S. A. Khrapak, ${ }^{2,3}$ i. Doğan, ${ }^{1}$ M. C. M. van de Sanden, ${ }^{1,4}$ and T. W. Morgan ${ }^{1}$ \\ ${ }^{1}$ DIFFER-Dutch Institute for Fundamental Energy Research, De Zaale 20, 5612 AJ Eindhoven, \\ The Netherlands \\ ${ }^{2}$ Aix Marseille University, CNRS, PIIM, Marseille, France \\ ${ }^{3}$ Joint Institute for High Temperatures, Russian Academy of Sciences, Moscow, Russia \\ ${ }^{4}$ Eindhoven University of Technology, PO Box 513, 5600 MB Eindhoven, The Netherlands
}

(Received 25 August 2017; accepted 6 October 2017; published online 7 November 2017)

In this study, we developed a revised stationary force balance model for particles in the regime $a / \lambda_{D}<10$. In contrast to other analytical models, the pressure and dipole force were included too, and for anisotropic plasmas, a novel contribution to the dipole moment was derived. Moreover, the Coulomb logarithm and collection cross-section were modified. The model was applied on a case study where carbon dust is formed near the plasma sheath in the linear plasma device Pilot-PSI. The pressure force and dipole force were found to be significant. By tracing the equilibrium position, the particle radius was determined at which the particle deposits. The obtained particle radius agrees well with the experimentally obtained size and suggests better agreement as compared to the unrevised model. Published by AIP Publishing. https://doi.org/10.1063/1.5001576

\section{INTRODUCTION}

Dust particles are observed in various plasma environments including space (e.g., in planetary rings and tails of comets), ${ }^{1}$ earth's ionosphere, ${ }^{2}$ as well as industrial and laboratory plasmas. In the latter case, dust formation is often an undesirable side-effect. For instance, dust particles can contaminate the synthesized thin films in plasma processing devices, ${ }^{3}$ cause reduction of the image quality in EUV lithography machines, ${ }^{4}$ and limit the performance and pose safety hazards in fusion reactors. ${ }^{5,6}$ On the other hand, nano-sized dust particles have also been shown to exhibit exotic properties, which can be used to study fundamental physical phenomena such as phase transitions ${ }^{7,8}$ or be employed in innovative materials. Examples of the latter application are biomarkers in tissue imaging, ${ }^{9}$ catalyzers for hydrogen production, ${ }^{10}$ charge storing elements in high-capacity batteries, ${ }^{11}$ and spectrum convertors in solar cells. ${ }^{12}$ These nanoparticles can effectively be synthesized by gas-phase aggregation in the plasma. ${ }^{13-15}$ In either case, whether desired or not, there is a strong need to predict/control the transport of these dust particles in plasma, which requires modelling of particle charge and forces.

In many of the above mentioned plasmas, typically the Debye length $\left(\lambda_{D}\right)$ is on the order of $1-0.01 \mathrm{~mm}$, and is considerably larger than the particle radius $(a)$ of up to a few micron. ${ }^{16-19}$ This permits (at least for mono-energetic ions ${ }^{20}$ ) the use of the orbit-motion-limited (OML) theory ${ }^{21-23}$ and neglection of particle screening. ${ }^{24}$ For some applications, however, this particle size limit no longer holds. For example, in magnetic fusion energy devices, ${ }^{25}$ the local Debye length is relatively small $(\sim 1 \mu \mathrm{m})$ due to the high density plasma $\left(>10^{20} \mathrm{~m}^{-3}\right)$, while the typical particle size can be up to tens of microns. ${ }^{6,26,27}$ Likewise, this is the case for dust particles that are injected in these plasmas on purpose. ${ }^{27-31}$ When the particle size approaches Debye length, the screening of the dust particles significantly affects the particle charge $\mathrm{e}^{24}$ and forces. ${ }^{16,32}$ Screening is, however, non-trivial to adequately include. This is due to the so-called absorption radius effect; ${ }^{24,33}$ for large particles, the particle potential profile exhibits barriers. These barriers result in reflection of a fraction of the incoming ions, which affects the charge distribution and thus the screening length of the particle. Most of the dust transport codes for magnetic fusion energy applications therefore neglect particle screening. In a recent work, ${ }^{24}$ it was shown that this can lead to an underestimation of the dust charge by an order of magnitude and may hence result in an incorrect description of dust transport (e.g., the escape fraction of dust towards the plasma core ${ }^{24}$ ), especially in the sheath region near the tokamak walls, where particles are repelled by the sheath electric field. ${ }^{34}$ This emphasizes the need to properly take into account particle screening.

Besides screening, particle forces which are typically neglected can become significant if the particle size approaches the Debye length. First of all, the dipole force may become dominant for increasing particle sizes as the dipole moment increases with the particle volume $\left(\sim a^{3}\right) .{ }^{16}$ Several contributions to the dipole moment can be present, e.g., due to the presence of an external field, ${ }^{32}$ and/or directed charging, ${ }^{35}$ and can mutually compete. Second, the polarization force, which arises due to deformation of the sheath in a density gradient, becomes significant. ${ }^{16}$ These forces have so far not yet been included in current dust transport codes for magnetic fusion energy devices. ${ }^{28,36-40}$

In this work, we will explore the consideration for the relevant particle charge and force equations in the situations $a \ll \lambda_{D}$ and $a<10 \lambda_{D}$ in Sec. II. Next, we will apply the obtained analytical description to one case study of interest - the growth of dust particles in the plasma sheath ${ }^{41}$ where the Debye length is shorter than the particle size. In Sec. III, a particle-plasma model is constructed, which is 
employed to determine the dominant forces as well as to explain the particle deposition mechanism and size distribution. The results are discussed and the conclusions are drawn in Sec. IV.

\section{THEORY}

\section{A. Charge and force equations for small particle sizes $\left(a \ll \lambda_{D}\right)$}

\section{Particle charging}

We review first the case where $a \ll \lambda_{D}$, and then Sec. II B indicates the changes that are required for $a<10$ $\lambda_{D}$. The Debye length we use in this work is linearized to take into account that ions cannot participate in the screening at suprathermal flows ${ }^{42}$

$$
\lambda_{D}=\lambda_{\text {lin }}=\left(\lambda_{D, i}{ }^{-2}\left(1+u^{2}\right)^{-1}+\lambda_{D, e}{ }^{-2}\right)^{-1 / 2},
$$

where $\lambda_{D, i(e)}=\sqrt{\varepsilon_{0} k_{b} T_{e(i)} / n_{e(i)} e^{2}}, \varepsilon_{0}$ is the vacuum permittivity, $k_{b}$ is the Boltzmann constant, $T_{e(i)}$ is the electron (ion) temperature, $n_{e(i)}$ is the electron (ion) density, $e$ is the elementary charge and $\vec{u}=\overrightarrow{v_{i}} / v_{T i}$ the dimensionless speed, $v_{T i}=\sqrt{k_{b} T_{i} / m_{i}}$ the ion thermal velocity, and $m_{i}$ the ion mass. Moreover, we assume $\lambda_{D} \ll \Delta$ in which $\Delta$ is the interparticle distance. If $\lambda_{D}>\Delta$, collective interactions have to be taken into account, see, e.g., Ref. 43.

By applying these axioms, the charge of a dust particle in the plasma can be obtained by using the OML theory. First we have to consider the direct ion and electron fluxes, which for isotropic plasmas $(\vec{u}=0)$ can be expressed by

$$
\begin{gathered}
I_{e}=-\sqrt{8 \pi} a^{2} e n_{e} v_{T e} \exp \left(\frac{e \varphi_{s}}{k_{b} T_{e}}\right), \\
I_{i}=\sqrt{8 \pi} a^{2} e n_{i} v_{T i}\left(1-\frac{e \varphi_{s}}{k_{b} T_{i}}\right),
\end{gathered}
$$

in which $v_{T e}=\sqrt{k_{b} T_{e} / m_{e}}$ is the electron thermal velocity, $m_{e}$ the electron mass, and $\varphi_{s}$ is the negative floating potential.

Besides these direct electron and ion fluxes, there are also other possible charging mechanisms. There are several electron emission processes from the dust particle, including photoelectric, secondary electron, and thermionic emission. ${ }^{17}$ Electron emission effectively increases the net dust charge and this can, under certain conditions, even lead to positive charges. Photoelectron emission and secondary electron emission are mostly important in astrophysics under the presence of UV radiation and high-energetic electrons $(>100 \mathrm{eV})$, and are not further discussed here (see for instance Ref. 43). Thermionic emission depends on the surface temperature $T_{\text {surf }}$ and work function $W$ of the particle material and can commonly be expressed (for $\left.\varphi_{s}<0\right)$ by

$$
I_{\text {th }}=\frac{\left(4 \pi a k_{b} T_{\text {surf }}\right)^{2} e m_{e}}{h^{3}} \exp \left(-\frac{W}{k_{b} T_{\text {surf }}}\right),
$$

where $h$ is the Planck constant.

Lastly, charging can also be affected by phenomena such as streaming ions, ${ }^{44}$ ion-neutral collisions ${ }^{45}$ and electronimpact ionization. ${ }^{46}$ For ions with high streaming velocities $u_{i} \gg v_{T i}$ (such as in plasma sheaths), the thermal ion energy term $k_{b} T_{i}$ in the ion current equation [Eq. (3)] is replaced with the kinetic energy of the drifting ions $m_{i} u_{i}{ }^{2} / 2^{44}$

$$
I_{\mathrm{i}}=\pi a^{2} e n_{i} u_{i}\left(1-\frac{2 e \varphi_{s}}{m_{i} u_{i}^{2}}\right) .
$$

Ion-neutral collision reduces the kinetic energy of the ions and this enhances ion collection on the dust surface. The resulting additional current in isotropic conditions can be approximated by ${ }^{45}$

$$
I_{\mathrm{i}-\mathrm{n}}=\sqrt{8 \pi} a^{2} e n_{i} v_{T i} 0.1\left(\frac{e \varphi_{s}}{k_{b} T_{i}}\right)^{2} \frac{\lambda_{D}}{l_{\mathrm{i}-\mathrm{n}}},
$$

where $l_{\mathrm{i}-\mathrm{n}}$ is the mean free path for ion-neutral collisions. In a similar manner, ion collection can be enhanced by electronimpact ionization of neutrals close to the grain. ${ }^{46}$ The effect for hydrogen plasmas becomes significant when the electron temperature is sufficiently high $(>15 \mathrm{eV})$.

The floating potential of the particle $\varphi_{s}$ is determined by the flux balance condition

$$
I_{e}\left(\varphi_{s}\right)+I_{i}\left(\varphi_{s}\right)+I_{\mathrm{th}}+I_{\mathrm{i}-\mathrm{n}}\left(\varphi_{s}\right)=0
$$

and the charge of the dust particle can be calculated from the Debye-Hückel potential (valid for a $<\lambda_{D}$ )

$$
Q=4 \pi \varepsilon_{0} a\left(1+\frac{a}{\lambda_{D}}\right) \varphi_{s},
$$

which is the so-called Whipple approximation. ${ }^{44}$ In the limit $a / \lambda_{D} \rightarrow 0$, particle screening can be fully neglected and we can determine the charge by Coulomb's law applied to an electrically floating sphere in vacuum

$$
Q=4 \pi \varepsilon_{0} a \varphi_{s}
$$

With the charge of the dust particle known, we can determine all relevant charge-dependent forces on the particle. These are summarized in Table I.

\section{Gravitational force}

The gravitational force can be expressed by

$$
\overrightarrow{F_{g}}=\frac{4}{3} \pi a^{3} \rho_{d} \vec{g}
$$

where $\rho_{d}$ is the mass density of the particle, and $\vec{g}$ is the gravitational acceleration.

\section{Neutral drag force}

The particles also experience a force of resistance from the surrounding medium-the neutral drag force. For most dusty plasmas, the relative velocity between the particle and neutral component $\vec{u}_{d}$ is much smaller than the thermal 
TABLE I. Two sets of charge and force equations that are used to obtain the critical particle size.

\begin{tabular}{lcc}
\hline \hline Force & Revised & Unrevised \\
\hline$\lambda$ & Eq. (1) & Eq. (20) \\
$Q$ & Eq. (8) & Eq. (8) \\
$F_{E}$ & Eq. (30) & Eq. (18) \\
$F_{\text {col }}$ & Eqs. (13) and (28) & Eq. (13) \\
$F_{\text {Coul }}$ & Eqs. (14), (24), and (29) & Eq. (14) \\
$F_{\text {dip,field }}$ & Eqs. (35) and (38) & $\ldots$ \\
$F_{\text {dip,ion }}$ & Eqs. (34) and (38) & $\ldots$ \\
$F_{\text {dip,sheath }}$ & Eqs. (37) and (38) & $\ldots$ \\
$F_{p}$ & Eq. (31) & $\ldots$ \\
\hline \hline
\end{tabular}

velocity $v_{T, n}$ of the neutrals, and the particle-gas system is in the free molecular regime (if the Knudsen number $K n \gg 1) .{ }^{43}$ In this situation, the neutral drag force can be written as

$$
\overrightarrow{F_{n}}=-\frac{8 \sqrt{2 \pi}}{3} \gamma a^{2} n_{n} T_{n} \frac{\vec{u}_{d}}{v_{T, n}},
$$

where $n_{n}$ and $T_{n}$ are the density and temperature of neutrals, respectively, and $\gamma$ is a coefficient on the order of unity. ${ }^{43}$

\section{Thermophoretic force}

In the presence of temperature gradient in the neutral gas, the particle will experience a force in the direction of lower temperatures. ${ }^{43}$ This so-called thermophoretic force can be expressed by

$$
\overrightarrow{F_{t h}}=-\frac{8 \sqrt{2 \pi}}{15} \frac{a^{2}}{v_{T}} \chi_{n} \nabla T,
$$

where $\chi_{n}$ is the thermal conductivity coefficient of gas. This coefficient can be estimated by $\chi_{n} \approx \frac{C \bar{v}}{\sigma_{t r}}$, where $\mathrm{C}$ is a numerical factor of the order of unity, $\bar{v} \stackrel{\sigma_{t r}}{=} \sqrt{8 T_{n} / \pi m_{n}}$, and $\sigma_{t r}$ is the transport scattering cross section for gas atoms or molecules. $^{43}$

\section{Ion drag force}

An analytical expression for the ion drag force of a single particle in collisionless Maxwellian plasmas is described in Ref. 42 . The ion drag force $\vec{F}_{i}$ is the sum of the collection part $\overrightarrow{F_{i, c o l}}$ (ions that directly impact on the particles) and the (Coulomb) scattering part $\overrightarrow{F_{i, \text { Coul }}}$ (momentum transfer to the particle from the ions which are scattered, but not collected)

$$
\begin{aligned}
\overrightarrow{F_{i, c o l}}= & \sqrt{2 \pi} a^{2} n_{i} m_{i} v_{T i}^{2}\left[\sqrt{\pi / 2} \operatorname{erf}\left(\frac{\vec{u}}{\sqrt{2}}\right)\right. \\
& \times\left[1+\vec{u}^{2}+\left(1-\vec{u}^{-2}\right)(1+2 \bar{z} \tau)\right] \\
& \left.+\vec{u}^{-1}\left[1+2 \bar{z} \tau+\vec{u}^{2}\right] \exp \left(-\frac{\vec{u}^{2}}{2}\right)\right],
\end{aligned}
$$

$$
\begin{aligned}
\overrightarrow{F_{i, \text { Coul }}}= & \sqrt{2 \pi} a^{2} n_{i} m_{i} v_{T i}^{2}\left[\sqrt{\pi / 2} \operatorname{erf}\left(\frac{\vec{u}}{\sqrt{2}}\right)\right. \\
& \left.\times\left[4 \bar{z}^{2} \tau^{2} \vec{u}^{-2} \ln \Lambda\right]-\vec{u}^{-1}\left[4 \bar{z}^{2} \tau^{2} \ln \Lambda\right] \exp \left(-\frac{\vec{u}^{2}}{2}\right)\right],
\end{aligned}
$$

in which $\vec{u}=\overrightarrow{v_{i}} / v_{T i}$ is the dimensionless ion speed, $\tau$ $=T_{e} / T_{i}, \bar{z}=e \varphi_{s} / T_{e}$, and where the Coulomb logarithm is defined as

$$
\ln \Lambda=\ln \left[\frac{\beta+1}{\beta+\frac{a}{\lambda_{D}}}\right]
$$

and

$$
\beta=\frac{\bar{z} \tau a}{\left(1+u^{2}\right) \lambda_{D}}
$$

is the scattering parameter, which is defined as the ratio of the interaction radius $r_{i n t}=U_{0} / m \vec{v}^{2}$ to the screening length $\lambda_{D}$-averaged over the ion velocities, where $\vec{v}$ is the relative velocity and

$$
U_{0}=e \varphi_{s} a e^{a / \lambda_{D}}
$$

is a potential energy constant based on the Debye-Hückel potential. $^{47}$ In the limit $\lambda_{D} \gg a$ (applied in this section), $U_{0}=e \varphi_{s} a$ [used in Eq. (16)] and $r_{\text {int }}=U_{0} / m \vec{v}^{2}$ equals the Coulomb radius $r_{C}$. $^{48}$

\section{Electric field force}

The electric field force on a particle with charge $Q$ can be expressed by ${ }^{16}$

$$
\overrightarrow{F_{e}}=Q \vec{E}
$$

in which $\vec{E}$ is the electric field vector.

Other forces including the rocket force, Lorentz force, electron drag, and radiation pressure force are not taken into account, as they are small compared to the dominant ion drag force. ${ }^{25}$

\section{B. Charge and force equations for large particle sizes $\left(a<10 \lambda_{D}\right)$}

In the case when the particle size exceeds the Debye length, the charge equations [Eqs. (8) and (9)] and force equations [Eqs. (10)-(14) and (18)] are in principle no longer valid. This is because the current collection to the particle transforms from spherical to planar geometry. If the particle size increases compared to the Debye length, this leads to a potential barrier $^{33}$ (and reflection) for ions with an impact parameter above a critical value, and thus an increasing fraction of ions that strike the particle head on. ${ }^{49}$ These ions are continuously accelerated, and their subsequent higher velocity reduces the ion density (because of flux conservation) and elongates the effective screening length. Furthermore, the potential barrier leads to a drop of the ion current towards the particle and subsequent rise of the floating potential. ${ }^{49,50}$ 


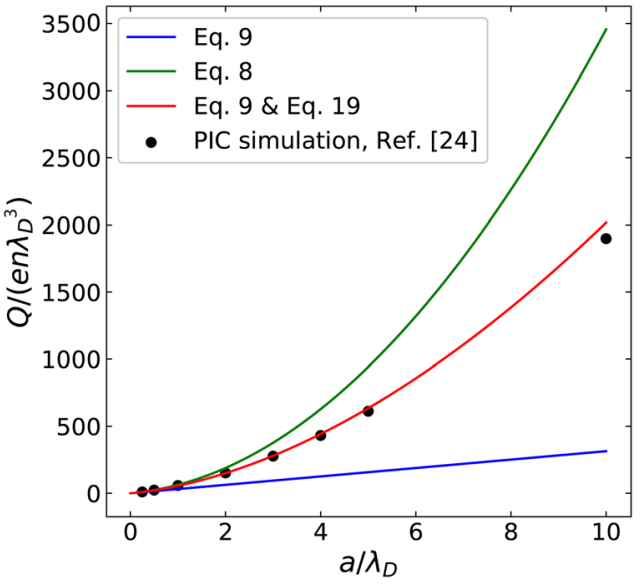

FIG. 1. The normalized charge as function of $a / \lambda_{D}$ by (a) neglecting screening, Eq. (8), (b) the Whipple approximation Eq. (9), (c) Eq. (9) in combination with the effective screening length, Eq. (19), and (d) results of a particle-in-cell simulation Ref. 24.

These two phenomena are not taken into account by the linearized Poisson equation which has been used to resolve the potential distribution (i.e., the Debye-Hückel potential). Hence, in principle, the full orbit motion theory has to be applied. ${ }^{33,51}$ In this section, we discuss an alternative approach. Moreover, we carefully revise the equations for the ion drag force and electric field force, and introduce the pressure (/polarization) and dipole force.

\section{Modification of effective screening length and particle charging}

Recent work ${ }^{52}$ shows that the exact non-linear solution of the Poisson equation near the particle can still be approximated by the linear Debye-Hückel (DH) potential if the linearized Debye length $\lambda_{D}$ is replaced by an effective screening length $\lambda_{s}>\lambda_{D}$. This effective screening length depends on the screening parameter $\bar{\beta}=\bar{z} \tau a / \lambda_{D, i}$ [same equation as Eq. (16) if $u=0$ and $\left.\lambda_{D}=\lambda_{D, i}\right]$ and can be expressed by ${ }^{52}$

$$
\lambda_{s, u=0}=\lambda_{D, i} \sqrt{1+0.48 \sqrt{\bar{\beta}}} .
$$

Although this equation was proposed for $a \leq 0.2 \lambda_{D}$ and $T_{i} \leq 0.1 T_{e}$, if implemented in the calculation of the charge $\left[\lambda_{D}\right.$ in Eq. (8) substituted for $\left.\lambda_{s, u=0}\right]$ it shows satisfactory

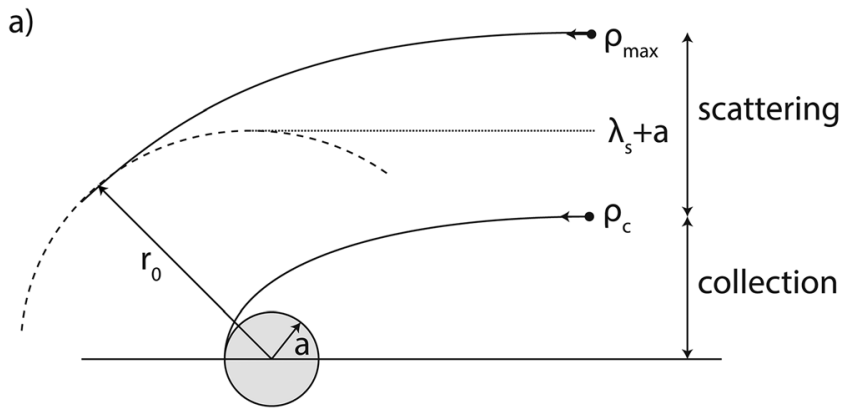

results as compared to the recent particle-in-cell (PIC) simulation results of Ref. 24. A significant improvement is achieved in comparison to not performing the substitution of $\lambda_{D}$ or neglecting screening [Eq. (9)]; see Fig. 1. This implies that Eq. (19) still holds for hydrogen plasmas and $T_{e} \approx T_{i}$. In the situation of drifting ions $(u>0)$, however, $\lambda_{s, u=0}$ may deviate. We propose a similar approach as used in Eq. (1), but substitute $\lambda_{D, i}$ for $\lambda_{s, u=0}$

$$
\lambda_{s}=\left(\lambda_{s, u=0}-2\left(1+u^{2}\right)^{-1}+\lambda_{D, e^{-2}}\right)^{-1 / 2} .
$$

In this way, the correct values for the effective screening length are obtained for the limits $u \rightarrow 0$ and $u \rightarrow \infty$. Throughout the remainder of the text, $\lambda_{s}$ was used as the effective screening length for the DB potential.

With regards to the increase of the floating potential, based on the aforementioned PIC simulation results, ${ }^{24}$ the transformation from spherical to planar current collection only results in a $12 \%$ increase of the floating potential for particle sizes up to $10 \lambda_{D}$ and $T_{i}=T_{e}$, and hence will be neglected.

\section{Modification of ion drag force}

In case shielding is taken into account, the Coulomb scattering part starts to diminish when $a$ approaches $\lambda_{D}$. Taken into consideration the significant particle size compared to the Debye length, we propose a modified lower and upper integration limit, $\rho_{\min }$ and $\rho_{\max }$, respectively, to calculate a revised Coulomb logarithm. As for the upper limit $\rho_{\max }$, all ions are taken into account with a distance of closest approach $r_{0}$ less than $\lambda_{s}+a$ (illustrated in Fig. 2), i.e., $r_{0}\left(\rho_{\max }\right)=\lambda_{s}+a$, rather than as $r_{0}\left(\rho_{\max }\right)=\lambda_{s}$ as in Ref. 53 . This results in the following expression for the upper limit:

$$
\rho_{\max }=\left(\lambda_{s}+a\right)\left(1+2 \beta_{s} \frac{\lambda_{s}}{\lambda_{s}+a}\right)^{1 / 2}
$$

where $\beta_{s}$ is obtained from Eq. (16) with $\lambda_{D}$ replaced by $\lambda_{s}$

$$
\beta_{s}=\frac{\bar{z} \tau a}{\left(1+u^{2}\right) \lambda_{s}} .
$$

The lower limit is the impact parameter for collection ${ }^{47}$

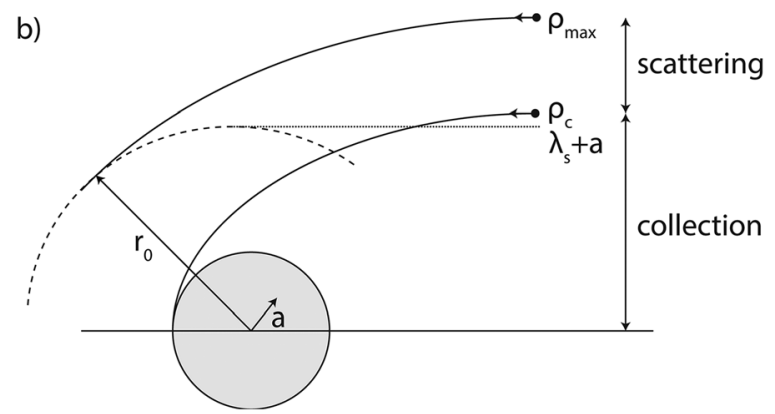

FIG. 2. Illustration of impacts of drifting $\mathrm{H}+$ ions on the surface of a dust particle. (a) In the typical situation $a \ll \lambda_{s}$ ions are collected with an impact parameter smaller than $\rho_{c}$, and are scattered in the range $\rho_{c}$ to $\rho_{\max }$, where the distance of closest approach is $r_{0}\left(\rho_{\max }\right)=\lambda_{s}+a$. (b) If the particle size becomes $a / \lambda_{s} \geq 1$, most of the ions are collected. 


$$
\rho_{\min }=\rho_{\mathrm{c}}=a\left(1+2 \beta_{s} \frac{\lambda_{s}}{a}\right)^{1 / 2} .
$$

Using Eqs. (21) and (23), the Coulomb logarithm can now be expressed by

$$
\ln \Lambda^{*}=\ln \left[\frac{\beta_{s}{ }^{2}+\rho_{\max }{ }^{2}}{\beta_{s}{ }^{2}+\rho_{\min }{ }^{2}}\right]^{1 / 2}=\ln \left[\frac{\beta_{s}+1+a / \lambda_{s}}{\beta_{s}+a / \lambda_{s}}\right] .
$$

It is evident that in the limit $a / \lambda_{s} \rightarrow \infty, \ln \Lambda=\ln [1]=0$. This gives some basis for a similar approach which was adopted in Ref. 54 to enforce non-negativity.

If $\beta_{s}>\beta_{c r}=13.2$, the limit of strong interaction holds, i.e., here the interaction radius is larger than the screening length. ${ }^{47}$ The screening parameter in this regime is defined as ${ }^{47}$

$$
\beta_{s}^{*}=\frac{\bar{z} \tau a e^{a / \lambda_{s}}}{\left(1+u^{2}\right) \lambda_{s}}=\beta_{s} e^{a / \lambda_{s}}
$$

because the $e^{a / \lambda_{s}}$ term in Eq. (17) becomes significant. In this regime, a potential barrier emerges ${ }^{48}$ if the ions have an impact parameter that exceeds the transitional impact parameter $\rho_{*}$, where

$$
\rho_{*}=\lambda_{D}\left(\ln \beta_{s}^{*}+1-\frac{1}{2} \ln ^{-1} \beta_{s}^{*}\right),
$$

with limiting value $\lim _{a / \lambda_{D} \rightarrow \infty} \rho_{*}=a$ and associated distance of closest approach $r_{\max } .{ }^{47}$ Due to the potential barrier the ions are reflected at much farther distances $\left(\gg \lambda_{s}\right)$. For $\rho<\rho_{*}$, no barrier exists. This separates the trajectories in two groups: far and close collisions, respectively. The cross section for Coulomb scattering comprises the sum of contributions from both groups $\sigma_{s}=\sigma_{s}^{\text {close }}+\sigma_{s}^{\text {far }}$, where

$$
\begin{aligned}
\sigma_{s}^{\text {close }} & =A \pi \rho_{*}{ }^{2} \\
\sigma_{s}^{\text {far }} & =B \lambda_{s}^{2}\left(1+2 \ln ^{-1} \beta_{s}^{*}\right)
\end{aligned}
$$

and $A=0.81$ and $B=6.4$, in the case of a point-like particle. $^{47}$ The impact parameter for collection becomes ${ }^{47}$

$$
\rho_{\mathrm{c}}=a\left(1+2 \beta_{s}^{*} \frac{\lambda_{s}}{a} e^{-a / \lambda_{s}}\right)^{1 / 2}
$$

and the associated cross section $\sigma_{c}=\pi \rho_{c}{ }^{2}$.

In the case of a hydrogen plasma and $T_{e}=T_{i}(\bar{z} \approx 2.5$, $\tau=1, u>1), \rho_{c}>\rho_{*}$ for $\beta_{s}^{*}>\beta_{c r}$. Because the ions with $\rho>\rho_{*}$ are reflected due to potential barrier and not absorbed, the impact parameter for collection reduces to $\rho_{*}$ (i.e., $\rho_{c}=\rho_{*}$ ), and the associated cross section becomes $\sigma_{c}=\pi \rho_{*}{ }^{2}{ }^{47}$ The collection part of the ion drag force for $\beta_{s}^{*}>\beta_{c r}$ can be approximated analogous to Ref. 55

$$
\overrightarrow{F_{i, C o l, \beta_{s}^{*}>\beta_{\mathrm{cr}}}},=\sigma_{c} n_{e} m_{i} v_{T i} \overrightarrow{v_{s}},
$$

where $\overrightarrow{v_{s}}=\sqrt{v_{T i}^{2}+{\overrightarrow{v_{s}}}^{2}}$ is the mean velocity of the ions. Concurrently, the cross section for close collisions $\left(\sigma_{s}^{\text {close }}\right)$ diminishes to zero because ions with $\rho<\rho_{*}$ are fully collected and not scattered. The ions with $\rho>\rho_{*}$ can, however, still lead to scattering by far collisions and thus contribute to $\sigma_{s}^{f a r}$. The associated scattering force can be approximated by

$$
\overrightarrow{F_{i, \text { Coul }, \beta_{s}^{*}>\beta_{\mathrm{cr}}, \mathrm{far}}}=\sigma_{s}^{\mathrm{far}} n_{e} m_{i} v_{T i} \overrightarrow{v_{s}} .
$$

If particle size $a$ exceeds the distance of closest approach associated with the potential barrier, $r_{\max }$, ions with $\rho_{c}>\rho$ $>\rho_{*}$ can still be absorbed rather than far scattered, i.e., $\sigma_{c}$ $=\pi \rho_{c}{ }^{2}$ in Eq. (28). The cross section for close-scattering remains zero and the fraction of ions that experience far collisions is reduced. This latter effects is not taken into account in current work, however. In the limit $a / \lambda_{D} \rightarrow \infty, \sigma_{s}^{\text {far }}$ becomes negligible and the total cross section for ion drag reduces to the geometrical cross section $\sigma_{\Sigma}=\sigma_{c, a / \lambda_{D} \rightarrow \infty}=\pi a^{2}$.

\section{Electric field, pressure, and polarization force}

In Ref. 56, it was shown that an external applied fieldfollowing the Debye-Hückel theory-causes polarization of the plasma surrounding the particle. Moreover, in Ref. 56, it is shown that in the case of a density gradient (e.g., in the sheath near the wall of a plasma device) the Debye length is spatially dependent which causes a deformation of the particle sheath. Both effects-assuming moment neutrality-induce polarization of the particle surface charge, which creates a field that exerts in turn an additional electrostatic force on the particle

$$
\begin{aligned}
\overrightarrow{F_{E}}= & Q \overrightarrow{E_{0}}\left[1+\frac{\left(a / \lambda_{s}\right)^{2}}{3\left(1+a / \lambda_{s}\right)}\right] \\
& -\frac{Q^{2}}{16 \pi \varepsilon_{0} \lambda_{s}^{2}}\left[\frac{\left(1+2 a / 3 \lambda_{s}\right)}{\left(1+a / \lambda_{s}\right)^{2}}\right] \frac{d \lambda_{s}}{d z} .
\end{aligned}
$$

In Ref. 56, it was shown that the external and particle field also exerts a force on the plasma. In fact, it increases the pressure force $F_{p}$ on the particle [terms up to $O\left(\lambda_{s} / L\right)^{3}$ ]

$$
\begin{aligned}
\overrightarrow{F_{p, s}} & =-Q \overrightarrow{E_{0}} \frac{\left(a / \lambda_{s}\right)^{2}}{3\left(1+a / \lambda_{s}\right)}+\frac{Q^{2}}{16 \pi \varepsilon_{0} \lambda_{s}{ }^{2}}\left[\frac{2 a / 3 \lambda_{s}}{\left(1+a / \lambda_{s}\right)^{2}}\right] \frac{d \lambda_{s}}{d z} \\
& -\frac{4}{3} \pi \mathrm{a}^{3} \frac{d p_{0}}{d z}+\frac{4}{3} \pi \mathrm{a}^{3} \rho_{0} \overrightarrow{E_{0}}-\frac{2}{15} \pi \mathrm{a}^{5} \frac{d^{3} p_{0}}{d z^{3}} \\
& +\frac{4}{5} \pi \frac{\mathrm{a}^{5}}{\lambda_{s}{ }^{3}} \varepsilon_{0} \overrightarrow{E_{0}} \frac{d \lambda_{s}}{d z} .
\end{aligned}
$$

In the common case $a \ll \lambda_{s}$ higher order terms $O\left(a / \lambda_{s}\right)^{3}$ can be neglected, i.e., only the first two terms are important. The sum of both contributions is then given by

$$
\vec{F}=Q \overrightarrow{E_{0}}-\frac{Q^{2}}{8 \pi \varepsilon_{0}} \frac{d \lambda_{s} / d z}{\left(\lambda_{s}^{2}+a^{2}\right)} .
$$

The first term equals the electrostatic force given by Eq. (18). The second term is called the polarization force

$$
\overrightarrow{F_{p o l}}=-\frac{Q^{2}}{8 \pi \varepsilon_{0}} \frac{d \lambda_{s} / d z}{\left(\lambda_{s}^{2}+a^{2}\right)} .
$$


In the case $a>\lambda_{s}$, the higher order terms of the pressure force cannot be neglected and will have to be taken into account [Eq. (31)].

\section{Dipole force}

The dipole force may become important in the presence of an electric field gradient and for increasing particle sizes (since it is proportional to the volume $\sim a^{3}$ ). ${ }^{16}$ The dipole moment can be induced in several ways.

First, it can arise due to anisotropy in charging. In Ref. 35 , the dipole moment induced by ion drift $\left(\overrightarrow{p_{\text {charge }}}\right)$ was calculated while neglecting screening. In the case of dielectric $\left(\varepsilon_{r} \neq \infty\right)$ particles, this can be expressed as

$$
\overrightarrow{p_{\text {charge }}}=+\frac{4 \pi \varepsilon_{0} a^{2} T_{e}}{e} \overrightarrow{U_{D}}(\vec{u}),
$$

where $\overrightarrow{U_{D}}(\vec{u})$ is the dimensionless dipole moment (in the opposite direction of $\vec{u}$ ), obtained by solving a set of nonlinear equations. $\overrightarrow{U_{D}}(\vec{u})$ is computed for the case of hydrogen up to $\vec{u}=6$ in Ref. 35 and is applied here. For $a>\lambda_{s}$, the impact parameter for collection reduces (similarly as in the case of the ion drag force), which may affect the dipole moment. This is, however, not taken into account in our work.

The dipole moment of a conducting particle $\left(\varepsilon_{r} \gg 1\right)$ induced by an external field (e.g., the sheath electric field) can be found by solving the Poisson equations self-consistently (see Ref. 32)

$$
\overrightarrow{p_{\text {field }}}=+4 \pi \varepsilon_{0} \overrightarrow{E_{0}} a^{3}\left[1+\frac{\left(a / \lambda_{s}\right)^{2}}{3\left(1+a / \lambda_{s}\right)}\right] .
$$

For dielectric particles, the dipole moment has to be multiplied by a factor $\xi=\left(\varepsilon_{r}-1\right) /\left(\varepsilon_{r}+2\right) .{ }^{57}$ In contrast to Ref. 57, we assume that the "apparent dipole moment" is equal to the dipole moment induced by an external field in vacuum:

$$
\overrightarrow{p_{\text {field }, \text { vac }}}=+4 \pi \varepsilon_{0} \xi \overrightarrow{E_{0}} a^{3} \text {. }
$$

This is consistent with assuming that the "apparent particle charge" is the charge calculated by OML, and the actual particle charge is $q_{0}=q\left(1+a / \lambda_{D}\right)$. The approach adopted in this paper is what Refs. 32 and 56 implicitly assumed.

Additionally, we introduce a novel induction mechanism for the dipole moment caused by deformation of the sheath due to the presence of a density gradient (similar situation as in Sec. II B 3). In Ref. 56, it was argued that the electric field and sheath-deformation induced dipole moment in the case of density gradient is zero. However, in this calculation of the dipole moment, the finite size $(a)$ of the particle was not taken into account. If this is considered (see the Appendix), the dipole moment becomes

$$
\overrightarrow{p_{\text {sheath }}}=-\frac{Q a^{3}}{4 \lambda_{D}{ }^{2}}\left[\frac{\left(1+2 a / 3 \lambda_{s}\right)}{\left(1+a / \lambda_{s}\right)^{2}}\right] \frac{d \lambda_{s}}{d z} .
$$

The total dipole force in the presence of anisotropic plasma $\left(\nabla \overrightarrow{E_{0}} \neq 0\right)$ and drifting ions can be calculated by including all aforementioned contributions of the dipole moment, and apply

$$
\overrightarrow{F_{d i p}}=\vec{p} \circ \nabla_{z} \overrightarrow{E_{0}}
$$

\section{CASE STUDY: DUST PARTICLES PRODUCED BY HIGH-FLUX PLASMA EXPOSURE}

Up until now, we have discussed the charge and force equations for the situation $a \ll \lambda_{D}$ and $a<10 \lambda_{D}$. In this section, we will apply these equations to one particular case study - carbon dust particle growth under high-flux hydrogen plasma exposure of graphite. In this situation, the source gas acting as growth precursor of the carbon dust particle is spontaneously created by chemically etching the graphite substrate. This leads to very rapid growth $(>0.4 \mu \mathrm{m} / \mathrm{s})$ of large particles $(\sim 1-5 \mu \mathrm{m})$ that exceed the Debye length $(\sim 1 \mu \mathrm{m})$ close to the target. The detailed experimental results and underlying growth mechanisms are presented in another article. $^{41}$

\section{A. Force balance model in the plasma sheath}

The particles are initially formed in the gas phase and are deposited at the surface during the discharge. Using the charge and force equations from Sec. II, we can now develop a stationary force model to identify the dominant forces on the particle.

In the plasma, we assume $\lambda_{D} \ll l_{\mathrm{i}-\mathrm{n}}$ and $\lambda_{D} \ll \Delta$ in which $l_{\mathrm{i}-\mathrm{n}}$ is the ion mean free path $(\sim 1 \mathrm{~mm}), \Delta$ is the inter-particle distance and $\lambda_{D}$ the linearized Debye length. Moreover, we neglect electron-emission processes; in Ref. 39, a coupled heating-charging model for dust particles (DUSTT) was applied which shows that the photoelectric, thermionic, and secondary electron emission are negligible in the case of our conditions $\left(T_{e} \approx T_{i}=1 \mathrm{eV}, n_{e}=4 \times 10^{20} \mathrm{~m}^{-3}\right)$. The effect of the ion-neutral collisions on the charging can be neglected because the mean free path of these collisions $\left(l_{\mathrm{i}-\mathrm{n}} \sim 1 \mathrm{~mm}\right)$ is much larger than the Debye length $\left(\lambda_{D} \sim 1 \mu \mathrm{m}\right)$. Moreover, the ion streaming effect on the charge (Sec. II A 1) was not taken into account as we assumed a constant charge as a function of $z$, the axial distance from the target. The effect of this assumption is discussed in Sec. III B. From the forces mentioned earlier, the gravitational force $\left(\rho_{g}=0.2-2 \mathrm{~g} / \mathrm{cm}^{3}\right)$ was found to be 4 orders of magnitude lower than the ion drag force for $a=1 \mu \mathrm{m}$. Moreover, the neutral friction and thermophoretic force were found to be about 2 orders of magnitude lower than the ion drag force. Therefore, the dominant forces are the electric field force, dipole force, polarization force, and ion drag force.

\section{Target sheath model}

In order for the particle to reach the surface, it has to go through the plasma sheath near the target. Therefore, the sheath conditions were modelled based on Ref. 58. For simplicity, we consider a neutral non-magnetized plasma with singly charged $\mathrm{H}$ ions in contact with an absorbing wall at floating potential. ${ }^{59}$ The ions are assumed to be mono-energetic $\left(T_{i}=0\right)$ and to 


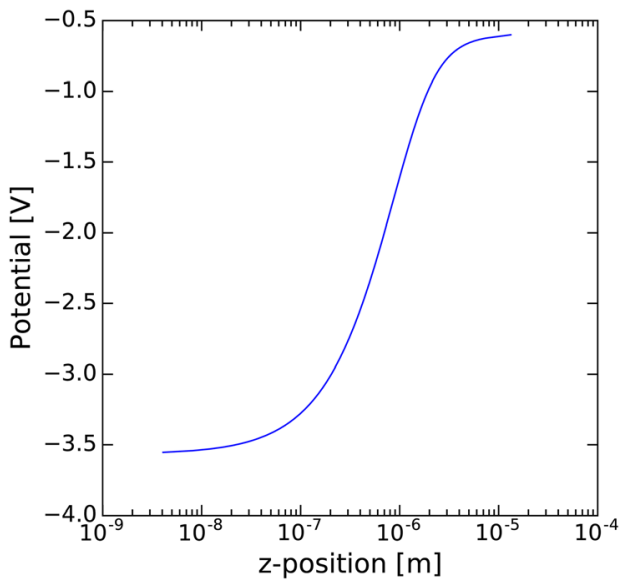

FIG. 3. Potential in the sheath as function of the distance to the target $(\mathrm{z}=0)$.

fall collisionlessly through the pre-sheath. ${ }^{58}$ In that case, the sheath is represented by the ion continuity, ion energy conservation, electron Boltzmann factor, and Poisson's equation as follows:

$$
\begin{gathered}
n_{i} v=n_{s e} v_{s e}, \\
\frac{1}{2} m_{i} v^{2}=\frac{1}{2} m_{i} v_{s e}^{2}-e \varphi, \\
n_{e}=n_{s e} \exp \left(-e \varphi / k_{b} T_{e}\right), \\
\frac{d^{2} \varphi}{d z^{2}}=-\frac{e}{\varepsilon_{0}}\left(n_{i}+n_{e}\right),
\end{gathered}
$$

where $n_{i}$ is the ion density, $v$ is the ion velocity, $n_{s e}=\frac{1}{2}$ $n_{\text {plasma }}$ is the ion density at the sheath-edge ( $n_{\text {plasma }}$ is the electron density in the plasma), ${ }^{58} v_{s e}=\sqrt{k_{b} T_{e} / m_{i}}$ (Bohm criterion), and $\varphi$ is the potential difference with the presheath. By substituting Eq. (39) in Eq. (40), the ion density can be expressed by

$$
n_{i}=n_{s e}\left(1+2 \varphi / m_{i} v_{s e}^{2}\right)^{-1 / 2}
$$

and by substituting this in Eq. (42), the following non-linear ODE is obtained:

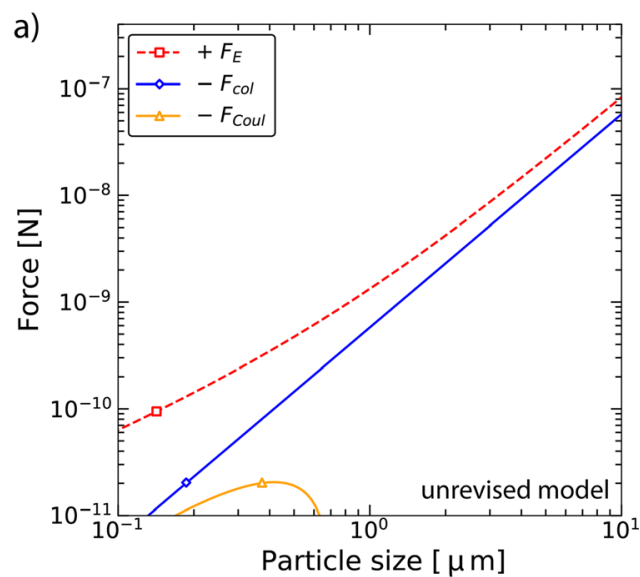

$$
\frac{d^{2} \varphi}{d z^{2}}=-\frac{e}{\varepsilon_{0}} n_{s e}\left(\left(1+2 \varphi / m_{i} v_{s e}^{2}\right)^{-1 / 2}+\exp \left(-e \varphi / k T_{e}\right)\right) .
$$

To solve the ODE, the boundary conditions of a fading potential distortion were applied ${ }^{59}$

$$
\varphi, \frac{\mathrm{d} \varphi}{\mathrm{dz}} \rightarrow 0 \quad \text { for } \mathrm{z} \rightarrow-\infty
$$

and we assumed the following potential drop from the presheath $V_{s e}=\frac{1}{2} k T_{e} / e:^{58}$

$$
\varphi(0) \approx-3 \frac{k T_{e}}{e}
$$

With these conditions, Eq. (44) was solved with a numerical solver. Assuming that the plasma potential is zero, the potential in the sheath becomes: $V_{s}=\varphi+V_{s e}$. The sheath potential is depicted in Fig. 3.

The potential exponentially decays towards the target with a decay length of $\sim 10 \lambda_{D}$. From this profile, the electric field is obtained by

$$
\vec{E}=-\frac{d \varphi}{d z} .
$$

In this way, the $z$-dependent parameters $v, n_{i}, d n / d z, \varphi$, $\vec{E}, d \vec{E} / d z$ were computed as function of the plasma parameters $n_{e}$ and $T_{e}$. In order to calculate the forces on the particles, we assume that outside the Debye length $\left(z>\lambda_{D, e}\right)$ $T_{i}=T_{e}$ and $n_{i}=n_{e}$ hold. In the case of a biased target, we used the boundary condition $V_{s}(0)=V_{\text {bias }}$ instead of Eq. (45).

\section{Evaluation of the force balance}

All the aforementioned equations are categorized per the scenario in Table I. The scenario "revised" is described by the revised equations in Sec. IIB, while the scenario "unrevised" refers to the unrevised equations described in Sec. II A. In Fig. 4, the forces of these equation sets are

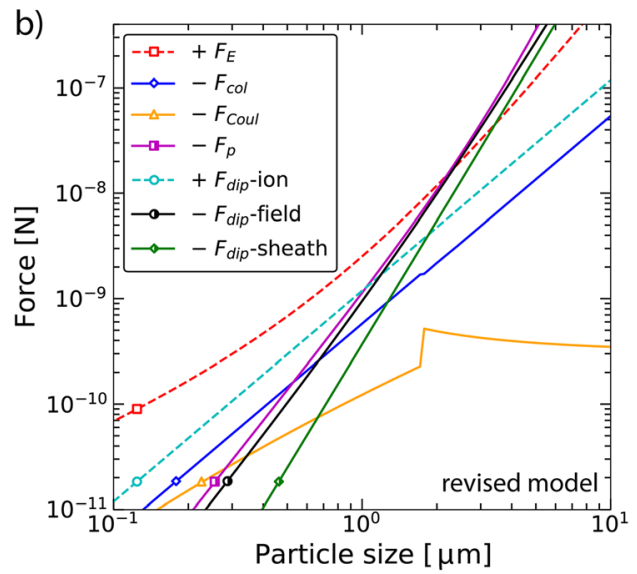

FIG. 4. The forces acting on the particle at $z=\lambda_{s}\left(n_{e}=4 \times 10^{20} \mathrm{~m}^{-3}, T_{e}=1.2 \mathrm{eV}\right)$ as a function of the particle size, including the $F_{\mathrm{E}}$ the electric field force, $F_{\text {col }}$ the ion collection force, $F_{\text {Coul }}$ the Coulomb scattering force, $F_{\text {dip }}$ the dipole force, and $F_{\mathrm{p}}$ pressure force. The graphs are based on the equations based on (a) "unrevised," and (b) "revised" model (see Table I). The "+" and "-" prefix indicate the direction of the force, pointing away from and towards the surface, respectively. 
depicted as a function of the particle radius at position $z=\lambda_{s}$, under plasma conditions $n_{e}=4 \times 10^{20} \mathrm{~m}^{-3}$ and $T_{e}=1.2 \mathrm{eV}$ (typical for Pilot-PSI ${ }^{60}$ ). It is apparent that the electric field force is initially dominant. With increasing particle size the ion drag force, pressure and dipole force eventually competes with the electric field force. The discontinuity of the Coulomb scattering force in Fig. 4(b) at $\mathrm{z}=2 \mu \mathrm{m}$ is due to the change in the model approach for the regime of weak and strong interactions (see Sec. II B 2). Consistently, the electric field and ion drag forces converge for small $a$ [Fig. S1(a) in the supplementary material].

In order to find the exact point of particle deposition, the equilibrium particle position (where $\min \left(\mathrm{z}\left[F_{\text {repulsive }}\right.\right.$ $\left.\left.>F_{\text {attractive }}\right]\right)$ ) was plotted as function of the particle radius in Fig. 5, for a floating target and a biased target at $-30 \mathrm{~V}$. The result shows that for an increasing particle size, the particle position (initially) moves closer to the surface, because the ion drag force becomes increasingly more significant. The discontinuous slope in the unrevised case is due to the nonnegativity condition of the Coulomb logarithm, Eq. (15). The sharp move to the surface at $a \sim 1 \mu \mathrm{m}$ can be explained by the rapid increase of the pressure force and the electric field and the sheath deformation induced dipole force [Eqs. (31), (35), and (37)]. Eventually, the particle reaches the surface when $z=a$ (dotted line) at the critical particle size $R_{\mathrm{c}}$. Consistently, the equilibrium position curves converge for small $a$ [Fig. S1(b) in the supplementary material]. The existence of a critical particle size is in line with Ref. 61, in which a time scan of the discharge duration shows that the surface is gradually being covered, while the mean size of the particles is not increasing significantly. The typical value for $R_{\mathrm{c}}$ obtained by our model under the aforementioned conditions is $\sim 1.1 \mu \mathrm{m}$, both for the floating as well as the biased target case. Neglecting the consideration on the particle screening and charging, as well as the ion drag, pressure and dipole force, results in a similar $R_{\mathrm{c}}$ in the case of a floating target, but to an overestimation of $R_{\mathrm{c}}$ by a factor 4.5 in the case of a biased target at $-30 \mathrm{~V}$.

\section{B. Discussion}

The dust particles synthesized in the experimental setup have been systematically analyzed by scanning and transmission electron microscopy in Ref. 41. The cross sectional images reveal that the particles contain a spherical core/ seed-i.e., grown in the gas phase-that predominantly consists of agglomerated nanoparticles, surrounded by accretion rings formed after deposition on the target. The typical size (radius) of the core is $\sim 1-5 \mu \mathrm{m}$. In comparison, the value for the critical particle size $R_{\mathrm{c}}$ found by our model under the condition of the experiment $\left(T_{e} \approx T_{i}=1 \mathrm{eV}\right.$, $\left.n_{e}=4 \times 10^{20} \mathrm{~m}^{-3}\right)$ is $\sim 1.1 \mu \mathrm{m}$, and is thus in agreement, i.e., $R_{\mathrm{c}}$ falls within the experimentally obtained range. Moreover, in an earlier work, ${ }^{62}$ it was found that the bulk particle size (core plus accretion rings) is equal for the floating and biased target case, which shows better consistency with our revised model than unrevised model. Nevertheless, we cannot exclude that this is not caused by factors currently not considered (e.g., an ion dependent etching rate).

In the calculation of the particle charge and forces we made several assumptions. First, we have assumed that the charge of the particle does not vary as function of the axial position and is equal to that at the sheath-presheath edge. Since the velocity increases towards the target, and the charging depends on the ion velocity due to the ion streaming effect [Eq. (5)], this could have led to an overestimation of the ion current up to a factor 2.7. Moreover, closer to the target, charge neutrality no longer holds $\left(n_{i}>n_{e}\right)$, which could result in up to $90 \%$ underestimation of the ion current [Eqs. (2) and (3)]. Second, we have neglected the effect of the magnetic field on the particle charging. Since under our conditions the particle size approaches the electron gyroratio length $r_{L, e}=\sqrt{m k_{b} T_{e}} / e B$, electrons start to move towards the particle in a straight line along the magnetic field line due to the gyromotion. This may reduce the electron current with a factor of up to 2 due to the transition from spherical to planar collection. $^{23,39,63}$ On the other hand, the ion current may also be reduced-for $a / r_{L, e}<10$ up to a factor $\sim 2$ - due to the creation of a potential hill. ${ }^{64,65}$ All these effects combined and taking into account the variation of the particle charge with the ion/electron current ratio (Fig. S2 in the supplementary material), the particle charge and floating potential may vary by a factor $0.6-1.3$.

The effect of the particle charge on the predicted critical particle size is depicted in Fig. 6. The general trend is that if the charge increases, the electric field repulsion from
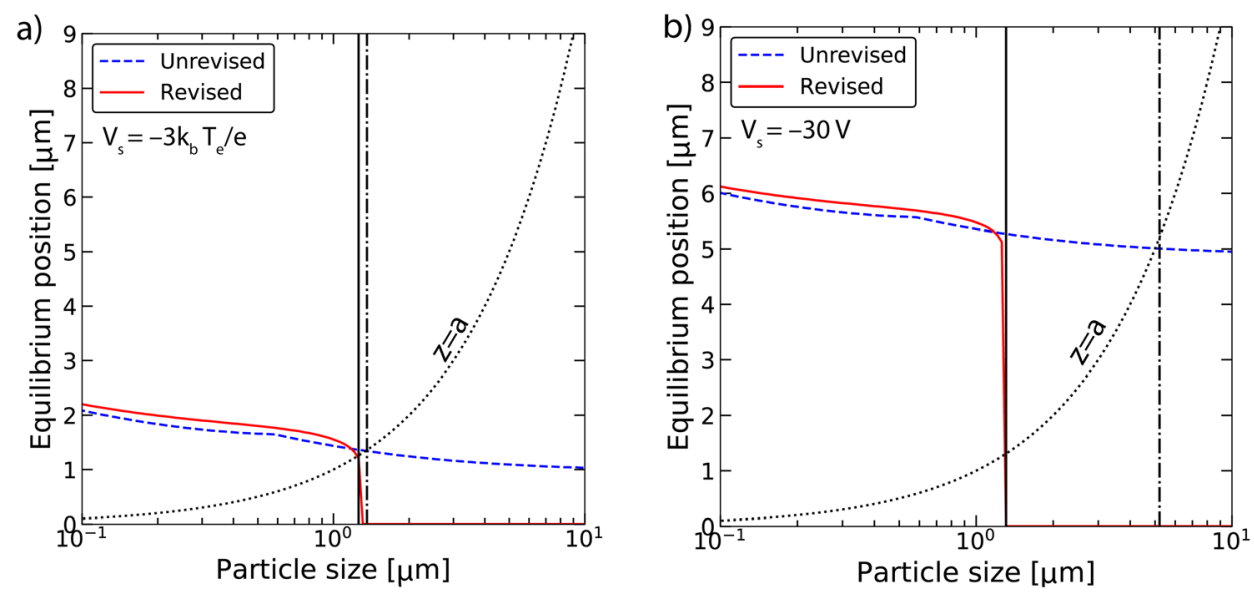

FIG. 5. The equilibrium position (in zdirection) of the particle as a function of the particle size obtained by using the equation sets of Table I, (a) floating potential: $-3 k_{b} T_{e} / e$ and (b) $-30 \mathrm{~V}$ bias. The black solid and dasheddotted vertical lines indicate at which particle size the particle deposits on the surface for the unrevised and revised cases, respectively. The particle is considered to deposit when the distance to the surface equals the particle radius: $z=a$ (dotted line). 


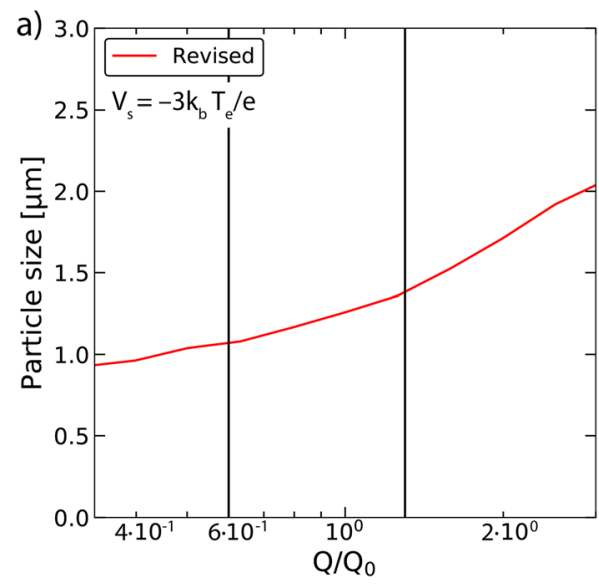

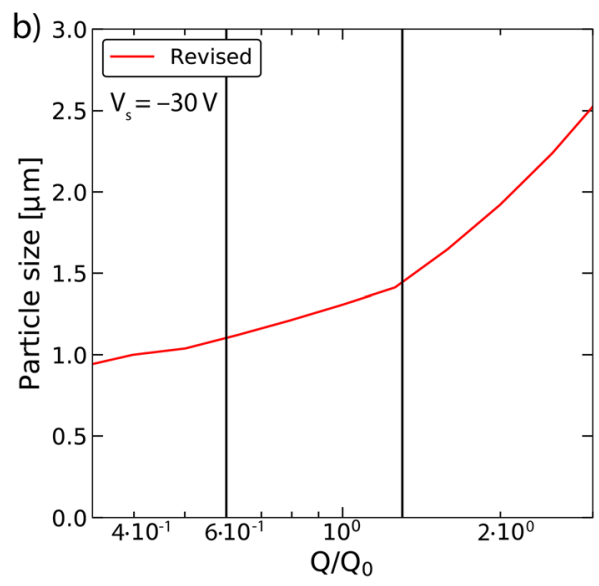

FIG. 6. Variation of the critical particle size as a function of the particle charge $\mathrm{Q} / \mathrm{Q}_{0}$ for (a) a floating target $\left(V_{s}=-3 k_{b} T_{e} / e\right)$ and (b) a biased target $\left(V_{s}=-30 \mathrm{~V}\right)$, respectively. In all graphs, the region between the vertical black lines shows the range of $Q / Q_{0}$. the surface rises compared to the attractive forces (e.g., the ion drag and pressure force), which leads to deposition at a larger critical particle size. Considering the maximum under/over-estimation of the charge, the critical particle size can be approximated within a variation of a factor 1.2.

Aforementioned effects such as by the magnetic field may besides affecting the value of the floating potential and charge also alter the shape (e.g., symmetry) of the potential distribution around the particle. This can have a significant impact on the ion drag, electrostatic, pressure and dipole forces, which are all based on the symmetrical form of the Debye-Hückel potential. Due to its complexity, this work falls outside the scope of current paper and is left for future research.

With regards to the experimental input into the model, the computed critical particle size may significantly vary as function of the plasma conditions $\left(\mathrm{n}_{\mathrm{e}}, \mathrm{T}_{\mathrm{e}}\right)$ during the experiments. Therefore, the critical particle size is presented as function $n_{e}$ and $T_{e}$ in Fig. 7 for a floating and biased target. The results show that the critical particle size increases with increasing $\mathrm{T}_{\mathrm{e}}$, which is related to the increase in particle charge and hence relative stronger repulsion from the surface (same effect as above). The decrease of the critical particle size with increasing $\mathrm{n}_{\mathrm{e}}$ is related to the relative increase of the attractive ion drag force. For better comparison, we included the plasma conditions during the experiments (scatter points), i.e., in this way, the predicted particle radius for the experimental settings can be determined. Given the variation in the target exposure conditions $\left(\sigma_{T_{e}} \approx 0.2 \mathrm{eV}, \sigma_{n_{e}}\right.$ $\left.\approx 1 \times 10^{20} \mathrm{~m}^{-3}\right)$, the critical particle size may vary less than $30 \%$.

Finally, we have only considered the axial force balance, and did not address other directions. From experiments, we observed that the dust deposition across the target is radially symmetric, which implies a negligible influence of lateral directed forces (i.e., the gravitational force) and the presence of a radial force balance. Indeed, due to the Gaussian shaped plasma beam profile in Pilot-PSI, radial electric fields and currents and density gradients are present, ${ }^{66}$ which result in similar particle forces (e.g., electric field, ion drag, and pressure forces) as in the axial direction. However, due to the radial symmetry these forces have no significant effect on the axial particle deposition behavior and are hence not further discussed.

\section{CONCLUSIONS}

This paper presents a heuristic stationary force balance model of dust particles in the plasma sheath for which the particle radius exceeds the Debye length. A correction for the ion drag force was presented and-in contrast to other more extensive models-the pressure and dipole force were included and for anisotropic plasmas a novel contribution to the dipole moment was derived. Moreover, the Coulomb logarithm and collection cross section were modified. A case study was investigated in which carbon dust particles are formed in the
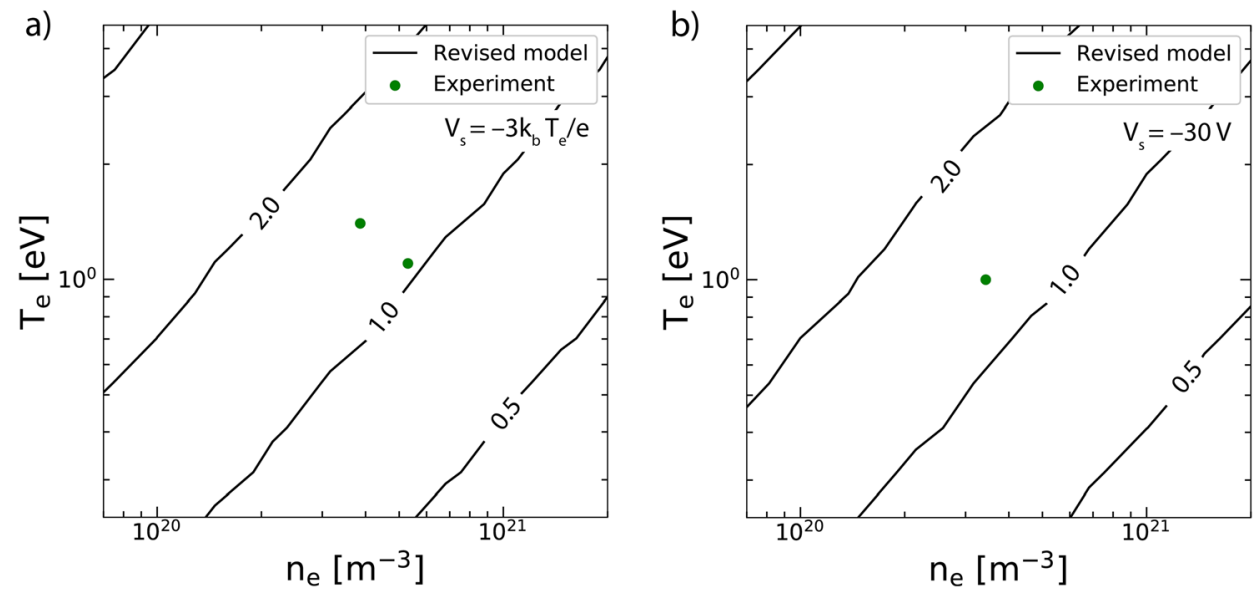

FIG. 7. Critical particle size (in $\mu \mathrm{m}$ ) as a function of the electron density $\left(\mathrm{n}_{\mathrm{e}}\right)$ and temperature $\left(\mathrm{T}_{\mathrm{e}}\right)$ for a target which is (a) floating $\left(V_{s}=-3 k_{b} T_{e} / e\right)$ and (b) biased $\left(V_{s}=-30 \mathrm{~V}\right)$. The scatter points in the plot show the central $(r=0)$ plasma conditions under which the targets are subjected to during the particle synthesis experiments. 
plasma sheath by high-flux plasma exposure on a graphite target. It was found that the pressure force and dipole force are significant in this situation. The particles can initially not reach the surface because they are charged negatively and are repelled by the electric field force in the sheath. Beyond a critical particle size, the ion drag force, pressure force, and dipole force will exceed the electric field force, however, and the particle is effectively pushed to the surface, mainly depending on the electron density and temperature. The critical particle size of deposition was derived by tracing the equilibrium position of the particle. The size calculated by this model is in fair agreement with the experimentally obtained particle size, and suggests better agreement as compared to the unrevised model.

\section{SUPPLEMENTARY MATERIAL}

See supplementary material for a more detailed comparison between the revised and unrevised model for the limit $a \ll \lambda_{D}$ and the variation of the particle charge with electron/ion flux ratio.

\section{ACKNOWLEDGMENTS}

This work is part of the research program of the Foundation for Fundamental Research on Matter (FOM), which is part of the Netherlands Organization for Scientific Research (NWO). S.A.K. was supported by the A*MIDEX Project (Nr. ANR-11-IDEX-0001-02) funded by the French Government "Investissements d'Avenir" Program managed by the French National Research Agency (ANR). The authors thank S. Ratynskaia for the discussions on charge and force equations for the regime in which the particle size exceeds the Debye length.

\section{APPENDIX: DERIVATION OF THE SHEATH- DEFORMATION INDUCED DIPOLE FORCE}

The dipole moment of the Debye shield around a finite size (a) particle can be computed by

$$
\overrightarrow{p_{s h}}=\int_{\mathrm{vol}} z \rho d V=2 \pi \int_{0}^{\pi} \int_{a}^{\infty} \rho_{s h}(r, \vartheta) r^{3} \cos \vartheta \sin \vartheta d r d \vartheta
$$

The charge is calculated using Poisson's equation $\Delta \Psi(r)$ $=-\rho(r) / \epsilon_{0}$, in which ${ }^{56}$

$$
\begin{aligned}
\Psi & =\Psi_{0}+\phi^{(0)}+\phi^{(1)}, \\
\Psi_{0}(z) & =\Psi_{0}(z)-\overrightarrow{E_{0}} z, \\
\phi^{(0)}(r) & =-\frac{Q \exp [-(r-a) / \lambda]}{4 \pi \varepsilon_{0}(1+a / \lambda) r}, \\
\phi^{(1)}(r) & =\overrightarrow{E_{0}}\left(\frac{a}{r}\right)^{3}\left(\frac{1+r / \lambda}{1+a / \lambda}\right) \exp [-(r-a) / \lambda] z,
\end{aligned}
$$

where $\lambda$ is the screening length

$$
-\frac{\sigma Q \lambda}{16 \pi \varepsilon_{0}(1+a / \lambda)}\left(\frac{a}{r}\right)^{3}\left(\frac{1+r / \lambda}{1+a / \lambda}-\left(\frac{r}{a}\right)^{3}\right) \exp [-(r-a) / \lambda] z,
$$

in which $\sigma \approx-2 \frac{1}{\lambda^{3}} d \lambda$. By using the relations (based on Ref. 56)

$$
\begin{gathered}
\Delta \varphi^{(0)}(r)=\frac{1}{\lambda^{2}} \varphi^{(0)}(r), \\
\Delta \varphi^{(1)}(r)=\frac{1}{\lambda^{2}} \varphi^{(1)}(r)+\sigma z \varphi^{(0)}(r),
\end{gathered}
$$

it follows that

$$
\rho(r, \vartheta)=\rho_{0}(r, \vartheta)+\rho_{s}(r, \vartheta),
$$

where $\rho_{s}(r)$ is the charge density of the Debye shield, given by

$$
\begin{aligned}
\rho_{s}(r, \vartheta)= & -\frac{\varepsilon_{0}}{\lambda^{2}(r)}\left(\phi^{(0)}(r, \vartheta)+\phi^{(1)}(r, \vartheta)\right) \\
& -\varepsilon_{0} \sigma z \phi^{(0)}(r, \vartheta) .
\end{aligned}
$$

Substituting this into Eq. (A1), and performing the integration results in the dipole moment of the Debye shield. Assuming moment neutrality $\left(\overrightarrow{p_{0}}=-\overrightarrow{p_{s h}}\right),{ }^{57}$ the dipole moment of the particle surface is

$\overrightarrow{p_{0}}=+4 \pi \varepsilon_{0} \overrightarrow{E_{0}} a^{3}\left[1+\frac{(a / \lambda)^{2}}{3(1+a / \lambda)}\right]-\frac{Q a^{3}}{4 \lambda_{D}^{2}}\left[\frac{(1+2 a / 3 \lambda)}{(1+a / \lambda)^{2}}\right] \frac{\mathrm{d} \lambda}{d z}$.

The first term is the same as Eq. (35) of this work [and equal to Eq. (18) of Ref. 32] and is attributed to the surface polarization induced by the electric field. The second term is attributed to the sheath deformation due to the density gradient and its resulting effect on the surface polarization. In the absence of plasma $(\lambda \rightarrow \infty)$, Eq. (A8) reduces to the dipole moment for a conducting particle $(\varepsilon \gg 1)$ in vacuum

$$
\overrightarrow{p_{0, v a c}}=4 \pi \varepsilon_{0} \overrightarrow{E_{0}} a^{3} \text {. }
$$

${ }^{1}$ C. K. Goertz, Rev. Geophys. 27, 271, https://doi.org/10.1029/ RG027i002p00271 (1989).

${ }^{2}$ O. Havnes, T. Aslaksen, and A. Brattli, Phys. Scr. T89, 133 (2001).

${ }^{3}$ K. G. Spears, T. J. Robinson, and R. M. Roth, IEEE Trans. Plasma Sci. 14 179 (1986).

${ }^{4}$ K. Takenoshita, C.-S. Koay, S. Teerawattansook, M. Richardson, and V. Bakshi, Proc. SPIE 5751, 563-571 (2005).

${ }^{5}$ J. Winter, Plasma Phys. Controlled Fusion 40, 1201 (1998).

${ }^{6}$ J. Winter, Plasma Phys. Controlled Fusion 46, B583 (2004).

${ }^{7}$ O. S. Vaulina, S. V. Vladimirov, O. F. Petrov, and V. E. Fortov, Phys. Rev. Lett. 88, 245002 (2002)

${ }^{8}$ A. Piel, Plasma Phys. Controlled Fusion 59, 14001 (2017).

${ }^{9}$ F. Erogbogbo, K. T. Yong, I. Roy, G. X. Xu, P. N. Prasad, and M. T. Swihart, ACS Nano 2, 873 (2008).

${ }^{10}$ F. Erogbogbo, T. Lin, P. M. Tucciarone, K. M. Lajoie, L. Lai, G. D. Patki, P. N. Prasad, and M. T. Swihart, Nano Lett. 13, 451 (2013).

${ }^{11}$ T. Kobayashi, Y. Imade, D. Shishihara, K. Homma, M. Nagao, R. Watanabe, T. Yokoi, A. Yamada, R. Kanno, and T. Tatsumi, J. Power Sources 182, 621 (2008). 
${ }^{12}$ J.-W. Luo, P. Stradins, and A. Zunger, Energy Environ. Sci. 4, 2546 (2011).

${ }^{13}$ I. Doğan, N. J. Kramer, R. H. J. Westermann, K. Dohnalová, A. H. M. Smets, M. A. Verheijen, T. Gregorkiewicz, and M. C. M. van de Sanden, J. Appl. Phys. 113, 134306 (2013).

${ }^{14}$ Y. Huttel, Gas-Phase Synthesis of Nanoparticles (Wiley-VCH Verlag $\mathrm{GmbH} \&$ Co. KGaA, 2017).

${ }^{15}$ P. V. Kashtanov, B. M. Smirnov, and R. Hippler, Physics-Uspekhi 50, 455 (2007).

${ }^{16}$ A. Bouchoule, Dusty Plasmas: Physics, Chemistry, and Technological Impact in Plasma Processing (Wiley, New York, 1999).

${ }^{17}$ V. E. Fortov and G. E. Morfill, Complex and Dusty Plasmas (CRC Press, Boca Raton, 2010).

${ }^{18}$ P. K. Shukla and A. A. Mamun, Introduction to Dusty Plasma (IOP Publishing Ltd., Bristol, 2002).

${ }^{19}$ S. A. Khrapak, A. V. Ivlev, G. E. Morfill, and H. M. Thomas, Phys. Rev. E 66, 046414 (2002).

${ }^{20}$ J. E. Allen, B. M. Annaratone, and U. De Angelis, J. Plasma Phys. 63, 299 (2000).

${ }^{21}$ H. M. Mott-Smith and I. Langmuir, Phys. Rev. 28, 727 (1926).

${ }^{22}$ Y. L. Al'pert and H. H. Nickle, Science 150, 875 (1965).

${ }^{23}$ J. E. Allen, Phys. Scr. 45, 497 (1992).

${ }^{24}$ G. L. Delzanno and X. Z. Tang, Phys. Plasmas 22, 113703 (2015).

${ }^{25}$ S. I. Krasheninnikov, R. D. Smirnov, and D. L. Rudakov, Plasma Phys. Controlled Fusion 53, 083001 (2011).

${ }^{26}$ J. P. Sharpe, D. A. Petti, and H. W. Bartels, Fusion Eng. Des. 63-64, 153 (2002).

${ }^{27}$ D. L. Rudakov, A. Litnovsky, W. P. West, J. H. Yu, J. A. Boedo, B. D. Bray, S. Brezinsek, N. H. Brooks, M. E. Fenstermacher, M. Groth et al., Nucl. Fusion 49, 085022 (2009).

${ }^{28}$ S. Ratynskaia, L. Vignitchouk, P. Tolias, I. Bykov, H. Bergsåker, A. Litnovsky, N. den Harder, and E. Lazzaro, Nucl. Fusion 53, 123002 (2013).

${ }^{29}$ A. Litnovsky, D. L. Rudakov, S. Bozhenkov, R. D. Smirnov, S. Ratynskaia, H. Bergsåker, I. Bykov, N. Ashikawa, G. De Temmerman, Y. Xu et al., J. Nucl. Mater. 438, S126 (2013).

${ }^{30}$ S. Ratynskaia, P. Tolias, A. Shalpegin, L. Vignitchouk, M. D. Angeli, I. Bykov, K. Bystrov, S. Bardin, F. Brochard, D. Ripamonti, N. den Harder, and G. D. Temmerman, J. Nucl. Mater. 463, 877 (2015).

${ }^{31}$ X. Z. Tang and G. L. Delzanno, J. Fusion Energy 29, 407 (2010).

${ }^{32}$ J. E. Daugherty, R. K. Porteous, and D. B. Graves, J. Appl. Phys. 73, 1617 (1993).

${ }^{33}$ I. B. Bernstein and I. N. Rabinowitz, Phys. Fluids 2, 112 (1959).

${ }^{34}$ S. I. Krasheninnikov, Y. Tomita, R. D. Smirnov, and R. K. Janev, Phys. Plasmas 11, 3141 (2004).

${ }^{35}$ A. V. Ivlev, G. Morfill, and V. E. Fortov, Phys. Plasmas 6, 1415 (1999).

${ }^{36}$ A. Y. Pigarov, S. I. Krasheninnikov, T. K. Soboleva, and T. D. Rognlien, Phys. Plasmas 12, 122508 (2005).

${ }^{37}$ J. D. Martin, M. Bacharis, M. Coppins, G. F. Counsell, and J. E. Allen, EPL 83, 65001 (2008).

${ }^{38}$ M. Bacharis, M. Coppins, and J. E. Allen, Phys. Rev. E 82, 026403 (2010).

${ }^{39}$ R. D. Smirnov, A. Y. Pigarov, M. Rosenberg, S. I. Krasheninnikov, and D. A. Mendis, Plasma Phys. Controlled Fusion 49, 347 (2007).
${ }^{40}$ L. Vignitchouk, P. Tolias, and S. Ratynskaia, Plasma Phys. Controlled Fusion 56, 95005 (2014).

${ }^{41}$ D. U. B. Aussems, K. Bystrov, I. Dogan, C. Arnas, M. Cabié, T. Neisius, M. Rasinski, E. Zoethout, P. Lipman, M. C. M. van de Sanden, and T. W. Morgan, Carbon 124, 403 (2017).

${ }^{42}$ S. A. Khrapak, A. V. Ivlev, S. K. Zhdanov, and G. E. Morfill, Phys. Plasmas 12, 042308 (2005).

${ }^{43}$ V. E. Fortov, A. G. Khrapak, S. A. Khrapak, V. I. Molotkov, and O. F. Petrov, Uspekhi Fiz. Nauk 174, 495 (2004).

${ }^{44}$ E. C. Whipple, Reports Prog. Phys. 44, 1197 (1981).

${ }^{45}$ S. A. Khrapak, S. V. Ratynskaia, A. V. Zobnin, A. D. Usachev, V. V. Yaroshenko, M. H. Thoma, M. Kretschmer, H. Höfner, G. E. Morfill, and O. F. Petrov, Phys. Rev. E 72, 016406 (2005).

${ }^{46}$ S. A. Khrapak and G. E. Morfill, Phys. Plasmas 19, 024510 (2012).

${ }^{47}$ S. A. Khrapak, A. V. Ivlev, G. E. Morfill, and S. K. Zhdanov, Phys. Rev. Lett. 90, 225002 (2003).

${ }^{48}$ M. D. Kilgore, J. E. Daugherty, R. K. Porteous, and D. B. Graves, J. Appl. Phys. 73, 7195 (1993).

${ }^{49}$ J. E. Daugherty, R. K. Porteous, M. D. Kilgore, and D. B. Graves, J. Appl. Phys. 72, 3934 (1992).

${ }^{50}$ C. T. N. Willis, M. Coppins, M. Bacharis, and J. E. Allen, Plasma Sources Sci. Technol. 19, 65022 (2010).

${ }^{51}$ J. G. Laframboise, "Theory of spherical and cylindrical Langmuir probes in a collisionless, Maxwellian plasma at rest," No. UTIAS-100, Institute for Aerospace Studies, University of Toronto, 1966.

${ }^{52}$ I. L. Semenov, S. A. Khrapak, and H. M. Thomas, Phys. Plasmas 22, 053704 (2015).

${ }^{53}$ S. A. Khrapak, M. Chaudhuri, and G. E. Morfill, IEEE Trans. Plasma Sci. 37, 487 (2009).

${ }^{54}$ I. H. Hutchinson and L. Patacchini, Plasma Phys. Control. Fusion 52, 124005 (2010).

${ }^{55}$ M. S. Barnes, J. H. Keller, J. C. Forster, J. A. O’Neill, and D. K. Coultas, Phys. Rev. Lett. 68, 313 (1992).

${ }^{56}$ S. Hamaguchi and R. T. Farouki, Phys. Plasmas 1, 2110 (1994).

${ }^{57}$ Y. Qizheng and T. Dan, J. Phys. D. Appl. Phys. 40, 4836 (2007).

${ }^{58} \mathrm{P}$. C. Stangeby, The plasma boundary of magnetic fusion devices (IOP Publishing Ltd., Bristol, 2000).

${ }^{59}$ K.-U. Riemann, J. Phys. D. Appl. Phys. 24, 493 (1991).

${ }^{60}$ G. J. Van Rooij, V. P. Veremiyenko, W. J. Goedheer, B. De Groot, A. W. Kleyn, P. H. M. Smeets, T. W. Versloot, D. G. Whyte, R. Engeln, D. C. Schram, and N. J. L. Cardozo, Appl. Phys. Lett. 90, 121501 (2007).

${ }^{61}$ K. Bystrov, L. van der Vegt, G. De Temmerman, C. Arnas, and L. Marot, J. Vac. Sci. Technol. A 31, 011303 (2013).

${ }^{62}$ K. Bystrov, M. C. M. Van De Sanden, C. Arnas, L. Marot, D. Mathys, F. Liu, L. K. Xu, X. B. Li, A. V. Shalpegin, and G. De Temmerman, Carbon 68, 695 (2014).

${ }^{63}$ L. Patacchini, I. H. Hutchinson, and G. Lapenta, Phys. Plasmas 14, 062111 (2007).

${ }^{64}$ L. Vignitchouk, S. Ratynskaia, and P. Tolias, Plasma Phys. Control. Fusion 59, 104002 (2017).

${ }^{65}$ J. R. Sanmartin, Phys. Fluids 13, 103 (1970).

${ }^{66}$ A. E. Shumack, H. J. de Blank, J. Westerhout, and G. J. van Rooij, Plasma Phys. Control. Fusion 54, 125006 (2012). 